Eur. J. Clin. Chem. Clin. Biochem.

Vol. 31, 1993, pp. 839-850

(C) 1993 Walter de Gruyter \& Co.

Berlin - New York

\title{
Complex Analyte-Dependent and Analyte-Independent Interferences with Conjugated Bilirubin in the Enzymatic Phenol-Aminophenazone Peroxidase (PAP) Method for Creatinine Determination
}

\author{
By C. D. Eng, R. Delgado and M. H. Kroll \\ Clinical Pathology Department, Warren G. Magnuson Clinical Center, National Institutes of Health, Bethesda, \\ $M D, U S A$
}

(Received February 22/July 14, 1993)

Summary: Although bilirubin interferes with the enzymatic assays for creatinine, neither a consensus of the degree of interference nor the mechanism has been established. Using multiple regression analysis, we demonstrate that the interference is negative and caused by both analyte-dependent and analyte-independent mechanisms. Furthermore, the correlative model includes terms non-linear with respect to creatinine. In the kinetic creatinine phenol-aminophenazone peroxidase method, there are analyte-dependent and analyteindependent mechanisms at work. The multivariate equation is: Crea' $=0.9879$ Crea -0.4524 Bili 0.000828 Crea $\times$ Bili $+2.094 \times 10^{-7} \mathrm{Crea}^{2} \times$ Bili $+5.0($ Crea' $=$ measured creatinine $(\mu \mathrm{mol} / \mathrm{l})$, Crea $=$ true creatinine $(\mu \mathrm{mol} / \mathrm{l})$, and Bili $=$ conjugated bilirubin $(\mu \mathrm{mol} / \mathrm{l}))$. The endpoint mode was affected less than the kinetic mode and exhibited different relationships in which two models describe the interference equally well. One is strictly analyte-dependent: $\mathrm{Crea}^{\prime}=0.9991 \mathrm{Crea}-0.00203 \mathrm{Crea} \times \mathrm{Bili}+2.390 \times 10^{-6} \mathrm{Crea}^{2}$ $\times$ Bili $-1.464 \times 10^{-9} \mathrm{Crea}^{3} \times$ Bili $+3.261 \times 10^{-13} \mathrm{Crea}^{4} \times$ Bili -9.9 . The other is a complex combined analyte-dependent and analyte-independent: $\mathrm{Crea}^{\prime}=0.9834 \mathrm{Crea}-0.00680 \mathrm{Crea} \times \mathrm{Bili}+2.477 \times 10^{-7}$

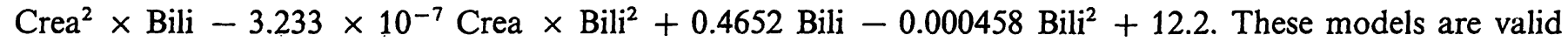
for creatinine concentrations up to $2200 \mu \mathrm{mol} / 1(24.9 \mathrm{mg} / \mathrm{dl})$ and bilirubin up to $660 \mu \mathrm{mol} / \mathrm{l}(38.6 \mathrm{mg} / \mathrm{dl})$. The interference increases with increments of either bilirubin or creatinine. In addition, we found that unconjugated bilirubin interferes differently from conjugated bilirubin in degree and mechanism. Model building, contour plots, surface plots, and possible mechanisms are discussed. We propose multiple regression analysis as the proper way to evaluate interferences because analyte-dependence can be easily missed by simple regression analysis. True creatinine concentrations can be estimated despite the interference from conjugated bilirubin. Other phenol-aminophenazone peroxidase methods may be similarly affected.

\section{Introduction}

Bilirubin is a well known negative interferent of the Jaffé method. There is some concern as to the degree of susceptibility for the phenol-aminophenazone peroxidase (PAP) method since compared with the Jaffe method in some studies $(1,2)$, the enzymatic assays for creatinine demonstrated less susceptibility to bilirubin and more as reported in others $(3,4)$. Moreover, published works suggest that conjugated bilirubin interferes more than unconjugated bilirubin in enzymatic assays $(5,6)$.
Our approach to interference studies is to derive a correlative equation by multiple regression analysis (7). The equation will contain terms which can be called analyte-dependent or analyte-independent. Analyte-independence is a term in which the degree of interference depends upon only the concentration of the interferent $(8,9)$. An example of this is pseudohyponatraemia is proportional only to the volume displacement caused by lipids. Another example is alkaline phosphatase, which when measured on the SMAC, is interfered by methotrexate because it ab- 
sorbs light at $340 \mathrm{~nm}$ like the product (10). Analytedependence, on the other hand, indicates that the interference depends upon the concentrations of both the interferent and the analyte. The dependence on the analyte does not necessarily mean that there is a reaction between the interferent and the analyte. More likely, there is a reaction, but not necessarily a chemical one, between the interferent and a product created by the reaction of the analyte and the reagents. The very nature of analyte-dependence implies non-linearity in an equation because of the cross-product term between the two. This concept is important because human intuition is poor in predicting responses in non-linear systems. The approach we have used will always detect the possibility of such interactions which simple regression fails to do. In addition, multivariate approach can handle systems where multiple interferents are not only present but which can interact among themselves as well as with the analyte or its products. This subject and its theoretical foundations have been discussed by Kroll \& Chesler (7).

In an earlier study, we developed a correlative model for the effect of unconjugated bilirubin on this assay. The effect was a complex analyte-dependent relationship without any evidence of analyte-independent mechanisms (11). Our aim is to explore the relationship of creatinine and conjugated bilirubin in the enzymatic creatinine assay. To our knowledge, neither a thorough quantitative analysis of the conjugated bilirubin interference in the enzymatic assay nor an analysis of the analyte-dependence of the interference has been done. In part this is because of the difficulty in obtaining conjugated bilirubin and its instability. Hence a synthetic conjugated bilirubin was used in this study. The literature supports the use of synthetic conjugated bilirubin as a substitute for the natural product $(5,6,12-14)$. Using multivariate regression (7-9), we demonstrated analyte-dependent and analyte-independent interferences. The derived equations are more complicated than the model to describe the interference from unconjugated bilirubin. Also discussed in this paper are the advantages of using multiple regression over standard regression, contour plots, surface plots, model building, and the possible mechanisms of interference will be addressed. Because this approach is still new, particular emphasis is placed on the model building and statistical aspects of this approach. Implications for other phenol-aminophenazone peroxidase based assays, such as for cholesterol, are mentioned. We believe the multivariate analysis approach is the proper method to examine interferences. There is no reason to assume that chemical interactions are linear over the broad range that exists in biochemical measurements of patients. In this research, we studied the enzymatic phenol-aminophenazone peroxidase method in the kinetic and endpoint modes. Our purpose is to seek further information on the mechanism of interference and not to compare the methods.

\section{Materials and Methods}

\section{Chemicals}

Creatinine was obtained from NIST (lot 914, Gaithersburg, MD). Synthetic ditaurine bilirubin derivative was obtained from Lee Scientific Inc. (lot C 64500, cat 910-10, St. Louis, $\mathrm{MO})$. A kit to analyze creatinine by the enzymatic phenol derivative-aminophenazone-peroxidase method was gotten from Boehringer Mannheim (cat 883263, Indianapolis, IN). When prepared according to the manufactuter's instructions, this kit contains solutions of the following concentrations:

Working Solution \#1: $4.3 \mathrm{mmol} / \mathrm{l}$ 4-aminophenazone, $>20000 \mathrm{U} / \mathrm{l}$ creatinase (EC 3.5.3.3; Pseudomonas species; $25^{\circ} \mathrm{C}$ ), > $12000 \mathrm{U} / \mathrm{l}$ sarcosine oxidase (EC 1.5.3.1; Pseudomonas species; $25^{\circ} \mathrm{C}$ ), $1000 \mathrm{U} / 1$ peroxidase (EC 3.1.1.13; horseradish; $25^{\circ} \mathrm{C}$ ), > $10000 \mathrm{U} / 1$ ascorbate oxidase (EC 1.10.3.3; Cucurbita species; $\left.25^{\circ} \mathrm{C}\right), 100 \mu \mathrm{mol} / 1$ potassium phosphate buffer (pH 7.9), $8.6 \mu \mathrm{mol} / 1$ 2,4,6-tribromo-3-hydroxybenzoic acid, $5 \mu \mathrm{mol} / \mathrm{l}$ potassium hexacyanoferrate(II), $3 \mathrm{~g} / \mathrm{l}$ detergent and

Working Solution \#2: (start reagent) $>164000 \mathrm{U} / 1$ creatininase (EC 3.5.2.10; Alcaligenes species; $25^{\circ} \mathrm{C}$ ), $20 \mu \mathrm{mol} / 1$ potassium phosphate buffer ( $\mathrm{pH} 7.9$ ), $3 \mathrm{~g} / \mathrm{l}$ detergent.

\section{Methods}

Creatininase converts creatinine to creatine; creatinase converts creatine to sarcosine and urea. Sarcosine oxidase converts sarcosine, water, and oxygen to glycine, formaldehyde, and hydrogen peroxide. The hydrogen peroxide reacts with modified Trinder reagents to produce a red chromophore whose absorbance is measured between 500 and $520 \mathrm{~nm}$. The reactions are as follows:

$\begin{array}{lll}\begin{array}{l}\text { Creatinine } \\ +\mathrm{H}_{2} \mathrm{O}\end{array} & \text { Creatininase } \\ \begin{array}{l}\text { Creatine } \\ +\mathrm{H}_{2} \mathrm{O}\end{array} & \text { Creatine } \\ \begin{array}{l}\text { Sarcosine }+\mathrm{H}_{2} \mathrm{O} \\ +\mathrm{O}_{2}\end{array} & \begin{array}{l}\text { Creatinase } \\ \text { Sarcosine oxidase }\end{array} & \begin{array}{l}\text { Sarcosine } \\ + \text { Urea } \\ +\mathrm{HCHO}+\mathrm{H}_{2} \mathrm{O}_{2}\end{array} \\ \begin{array}{l}\mathrm{H}_{2} \mathrm{O}_{2}+\text { Phenol } \\ \text { derivative }+\end{array} & \stackrel{\text { Peroxidase }}{\longrightarrow} & \begin{array}{l}\text { Red benzoquinone- } \\ \text { 4-Amine dye }\end{array}\end{array}$

\section{Instrumentation}

The Boehringer Mannheim Creatinine phenol-aminophenazone peroxidase kit can be adapted to several automated instruments as well as performed as a manual method. The Creatinine phenol-aminophenazone peroxidase method can be run in the endpoint or kinetic mode, both of which we used. Conditions were optimized for use on a Cobas Mira (Roche Diagnostic Systems, Nutley, NJ, USA) using aqueous standards. The settings we employed are in listed in table 1.

\section{Solutions}

\section{Creatinine}

By dissolving $50 \mathrm{mg}$ creatinine in $10 \mathrm{ml}$ of pooled serum, we made a $422 \mathrm{mmol} / \mathrm{l}$ stock solution. 
Tab. 1. Instrument settings

\begin{tabular}{lrr}
\hline Condition & Kinetic & Endpoint \\
\hline Wavelength $(\mathrm{nm})$ & 500 & 500 \\
Sample volume $(\mu \mathrm{l})$ & 7 & 5 \\
Sample dilucnt $(\mu \mathrm{l}$ water) & 30 & 35 \\
Reagent 1 volume $(\mu \mathrm{l})$ & 200 & 200 \\
Start time: rcagent 1 & & \\
$\quad$ (s into cycle) & 18 & 18 \\
$\quad$ (transfer/analysis cycle) & 1 & 1 \\
Preincubation time (s) & 257 & 257 \\
$\quad$ (transfer/analysis cycle) & 11 & 11 \\
Reagent 2 volume $(\mu \mathrm{l})$ & 35 & 40 \\
Reagent 2 diluent $(\mu \mathrm{l})$ & 20 & 20 \\
Start time: start reagent & 268 & 268 \\
$\quad$ (transfer/analysis cycle) & 11 & 11 \\
First reading (s) & 258 & 258 \\
$\quad$ (transfer/analysis cycle) & 11 & 11 \\
Last reading (s) & 507 & 1132 \\
$\quad$ (transfer/analysis cycle) & 20 & 45 \\
\hline
\end{tabular}

\section{Conjugated bilirubin}

In a dimly lit room, we dissolved $20 \mathrm{mg}$ of ditaurine bilirubin in $25 \mathrm{ml}$ of pooled serum, to produce a $13.68 \mathrm{mmol} / \mathrm{l}$ stock solution. We added varying volumes of the stock solution to a human serum pool that we had filtered three times using Whatman \# 1 filter paper, followed by a $0.45 \mu \mathrm{m}$, and then a 0.2 $\mu \mathrm{m}$ membrane to produce six different concentrations of bilirubin. Using the method of Jendrassik \& Grof (15), we measured the bilirubin for both methods at $6.8,80.4,160.7,326.6$, 458.3, and $656.6 \mu \mathrm{mol} / \mathrm{l}$ on the Hitachi 736 (Boehringer Mannheim. Indianapolis, IN).

\section{Construction of a sample matrix}

We created a matrix of samples in which the creatinine and bilirubin varied independently of one another $(7,9)$. The bilirubin concentration increased across the rows and the creatinine concentration increased down the columns. We divided each bilirubin pool into six aliquots and to each we added equal volumes of the pooled serum with added creatinine to produce six different concentrations, hence thirty-six specimens in all.
The concentrations of creatinine, before the interferent was added, measured $221,345,583,1043,1618$, and $2086 \mu \mathrm{mol} / \mathrm{l}$ for the endpoint mode and 221,345,592,1061, 1653, and 2175 $\mu \mathrm{mol} / \mathrm{l}$ for the kinetic mode.

\section{Results}

We set up the enzymatic phenol-aminophenazone peroxidase method according to standard methods optimizing the sample to reagent ratios and the measuring times of the assay for the Cobas Mira (tab. 1). The correlation between the measured creatinine and the creatinine standards were $y=0.986 x+0.09$ and $\mathrm{y}=1.02 \mathrm{x}+0.02(\mathrm{y}=$ measured creatinine; $\mathrm{x}=$ aqueous standard $\mathrm{mg} / \mathrm{dl}$ ) for the kinetic and endpoint

Tab. 2. Effects of conjugated bilirubin and creatinine on the creatinine phenol-aminophenazone peroxidase method

Bilirubin $(\mu \mathrm{mol} / \mathrm{l})^{*}$

$\begin{array}{llllll}6.8 & 80.4 & 160.7 & 326.6 & 458.3 & 656.6\end{array}$

(A) Creatinine by endpoint mode $(\mu \mathrm{mol} / \mathrm{l})$

$\begin{array}{rrrrrr}221 & 177 & 133 & 80 & 35.4 & 35.4 \\ 345 & 301 & 256 & 168 & 97.2 & 44.2 \\ 583 & 522 & 460 & 362 & 274 & 159 \\ 1043 & 955 & 928 & 796 & 663 & 522 \\ 1618 & 1520 & 1441 & 1299 & 1132 & 964 \\ 2086 & 1936 & 1936 & 1759 & 1697 & 1432\end{array}$

(B) Creatinine by kinetic mode $(\mu \mathrm{mol} / \mathrm{l})$

\begin{tabular}{rrrrcc}
221 & 159 & 115 & 35.3 & $* *$ & $* *$ \\
345 & 301 & 221 & 106 & 26.5 & $* *$ \\
592 & 504 & 442 & 292 & 150 & 35.4 \\
1061 & 937 & 919 & 743 & 530 & 354 \\
1653 & 1520 & 1450 & 1299 & 955 & 831 \\
2175 & 1998 & 1936 & 1724 & 1609 & 1326 \\
\hline
\end{tabular}

* The bilirubin concentration for each result is given in the top row.

** Absorbance too low for instrument to calculate a value

Tab. 3. Simple regression model

\begin{tabular}{llllllrrr}
\hline Method & $\begin{array}{l}\text { Creatinine } \\
(\mu \mathrm{mol} / \mathrm{l})\end{array}$ & Slope & $\begin{array}{l}\text { Intercept } \\
(\mu \mathrm{mol} / \mathrm{l})\end{array}$ & $\mathrm{r}^{2}$ & $\mathrm{SE}_{\text {slope }}$ & $\mathrm{MS}_{\mathrm{r}}$ & $\mathrm{t}-\mathrm{value}$ & $\mathrm{p}$ \\
\hline Endpoint & 221 & -0.2930 & 196 & 0.893 & 0.0508 & 787 & 5.8 & 0.0045 \\
& 345 & -0.4757 & 336 & 0.983 & 0.0311 & 294 & 15.3 & 0.0001 \\
& 583 & -0.6449 & 575 & 0.997 & 0.0180 & 99 & 35.8 & 0.0001 \\
& 1043 & -0.7956 & 1042 & 0.994 & 0.0311 & 294 & 25.6 & 0.0001 \\
& 1618 & -1.0001 & 1611 & 0.996 & 0.0305 & 284 & 32.8 & 0.0001 \\
& 2086 & -0.9209 & 2067 & 0.970 & 0.0805 & 1973 & 11.4 & 0.0003 \\
Kinetic & 221 & -0.564 & 213 & 0.983 & 0.052 & 155 & 10.8 & 0.0085 \\
& 345 & -0.7186 & 348 & 0.995 & 0.0295 & 118 & 24.3 & 0.0002 \\
& 592 & -0.8689 & 581 & 0.991 & 0.0411 & 514 & 21.1 & 0.0001 \\
& 1061 & -1.0881 & 1064 & 0.987 & 0.0633 & 1221 & 17.2 & 0.0001 \\
& 1653 & -1.3019 & 1651 & 0.967 & 0.1196 & 4357 & 10.9 & 0.0004 \\
& 2175 & -1.2288 & 2141 & 0.989 & 0.0645 & 1268 & 19.0 & 0.0001 \\
\hline
\end{tabular}

$\mathrm{r}^{2} \quad=$ coefficient of determination

$\mathrm{SE}_{\text {slope }}=$ standard error of the slope

$\mathrm{MS}_{\mathrm{r}}=$ mean square of the residuals

p = probability of the null hypothesis being true, i.e. the slope is not significantly different from zcro 
calculations, respectively. These methods are linear to at least $2210 \mu \mathrm{mol} / \mathrm{l}(25 \mathrm{mg} / \mathrm{dl})$. The kinetic assay had within-run coefficient of variations $(n=6)$ of $3.2 \%$ and $1.4 \%$ for the low (Precical) and high control (Precitrol), respectively. The coefficients of variation for the endpoint assay were $3.2 \%$ and $0.7 \%$ for the low and high control, respectively. The results for precision are similar to that reported in the literature $(16,17)$.

As can be seen from table 2, we tested a matrix in which both the concentrations of creatinine and conjugated bilirubin varied over a broad range. There are marked decreases in the measured creatinine as the concentration of conjugated bilirubin increased. The percentage drop is greater at lower concentrations of creatinine. The influence of the two components were analyzed by multivariate regression on a Macintosh $\mathrm{SE}$ using the program Stat View SE+ Graphics (Abacus Concepts Inc., 1988) and following the rules for analyzing analyte-dependent interferences. These methods are described by Kroll \& Chesler (7) and by Draper \& Smith (18).

The typical response of the assay to bilirubin for both the kinetic and endpoint methods appears to be a linear relationship with a negative slope (coefficient). A simple linear regression of the data in table 2 produces a different negative slope for each set of creatinine concentrations (tab. 3). Some of these slopes vary significantly from one another. Figure 1 shows plots of the slopes from table 3 plotted against the various starting creatinine concentrations. The best fitting curves for the endpoint and kinetic methods are

$$
\begin{aligned}
\frac{\mathrm{dCrea}}{\mathrm{dBili}} & =0.2215-0.003124 \mathrm{Crea} \\
& +4.043 \times 10^{-6} \mathrm{Crea}^{2} \\
& -2.451 \times 10^{-9} \mathrm{Crea}^{3} \\
& +5.299 \times 10^{-13} \mathrm{Crea}^{4} \\
& \left(\mathrm{r}^{2}=1.000\right)
\end{aligned}
$$

and

$$
\begin{aligned}
\frac{\mathrm{dCrea}}{\mathrm{dBili}} & =-0.3694-0.00103 \text { Crea } \\
& +2.912 \times 10^{-7} \mathrm{Crea}^{2} \\
& \left(\mathrm{r}^{2}=0.991\right)
\end{aligned}
$$

respectively. The equation:

$$
\begin{gathered}
\frac{\mathrm{dCrea}}{\mathrm{dBili}}=0.1276-0.00100 \mathrm{Crea} \\
+2.952 \times 10^{-7} \mathrm{Crea}^{2} \\
\left(\mathrm{r}^{2}=0.978\right)
\end{gathered}
$$

also fits the endpoint method. If the interferences were straightforward, that is, simple analyte-independence, the slopes would be zero (7-9).

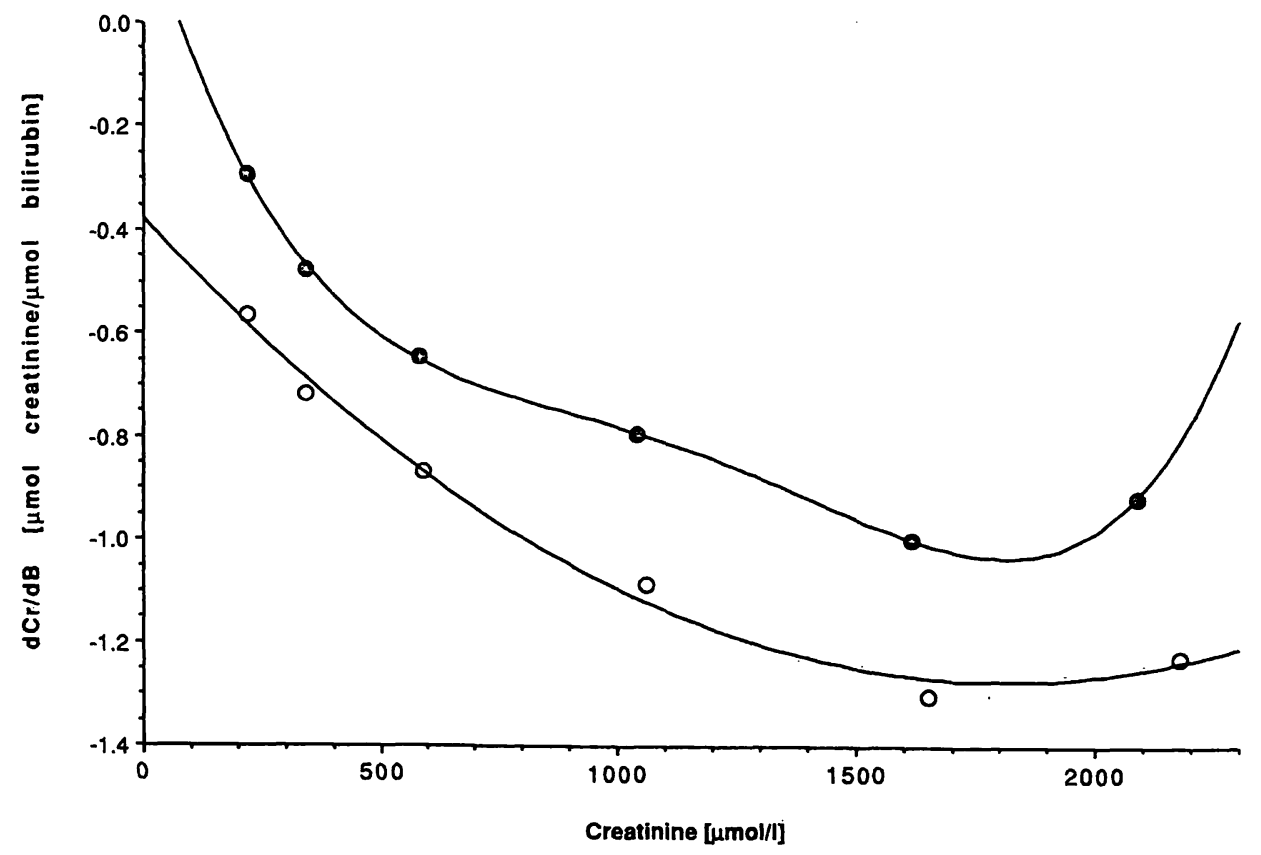

Fig. 1. Plot of slopes, $\frac{\mathrm{dCrea}}{\mathrm{dBili}}$, derived from simple regression analysis (tab. 3) vs. creatinine

- Endpoint Mode:

$\mathrm{dCrea} / \mathrm{dBili}=0.222-0.0031$ Crea $+4.04 \times 10^{-6} \mathrm{Crea}^{2}-2.45 \times 10^{-9} \mathrm{Crea}^{3}+5.30 \times 10^{-13} \mathrm{Crea}^{4}$

o Kinetic Mode:

$\mathrm{dCrea} / \mathrm{dBili}=-0.369-0.0010 \mathrm{Crea}+2.91 \times 10^{-7} \mathrm{Crea}^{2}$ 
A stepwise procedure was used to generate the multivariate models:

$$
\begin{aligned}
\text { Crea }^{\prime}=b_{00} & +b_{10} \text { Crea }+b_{01} \text { Bili } \\
& +b_{20} \text { Crea }^{2}+b_{11} \text { Crea Bili }+b_{02} \text { Bili }^{2} \\
& +b_{30} \text { Crea }^{3}+b_{21} \text { Crea }^{2} \text { Bili } \\
& +b_{12} \text { Crea Bili }^{2}+b_{03} \text { Bili }^{3} \\
& +b_{40} \text { Crea }^{4}+b_{31} \text { Crea }^{3} \text { Bili } \\
& +b_{22} \text { Crea }^{2} \text { Bili }^{2}+b_{13} \text { Crea Bili } \\
& +b_{04} \text { Bili }^{4}
\end{aligned}
$$

Tab. 4. Comparison of selected models

\begin{tabular}{lllll}
\hline Model & Degrees & Sum & Mean & F ratio: \\
& of & squares & square & alternative \\
freedom & of the & of the & model vs. \\
& (DF) & residuals & residuals & model with \\
& & (SS) & (MSr) & lowest MSr
\end{tabular}

Endpoint method

$\begin{array}{lllll}\text { a) } \begin{array}{l}2 \text { parameter } \\ \text { Crea }\end{array} & 33 & 143815 & 4358 & 77^{*} \\ \text { Crea } \times \text { Bili } & & & & \\ \text { b) } 2 \text { parameter } & 33 & 129954 & 3938 & 68 \\ \text { Crea } & & & & \\ \text { Bili } & & & & \\ \text { c) } 3 \text { parameter } & 32 & 51723 & 1616 & 21 \\ \text { Crea } & & & & \\ \text { Crea } \times \text { Bili } & & & & \\ \text { Bili } & & & \\ \text { d) } 6 \text { parameter } & 29 & 16800 & 579 & 0.04 \\ \text { Crea } & & & & \\ \text { Crea } \times \text { Bili } & & & & \\ \text { Crea } \times \text { Bili } \\ \text { Crea } \times \text { Bili } \\ \text { Bili, Bili } \\ \text { e) } & & & & \\ \text { parameter } & 30 & 16618 & 554 \\ \text { Crea } & & & \\ \text { Crea } \times \text { Bili } & & & \\ \text { Crea } \times \text { Bili } & & & \\ \text { Crea }{ }^{3} \times \text { Bili } & & & \\ \text { Crea } \times \text { Bili } & & & \end{array}$

Kinetic method

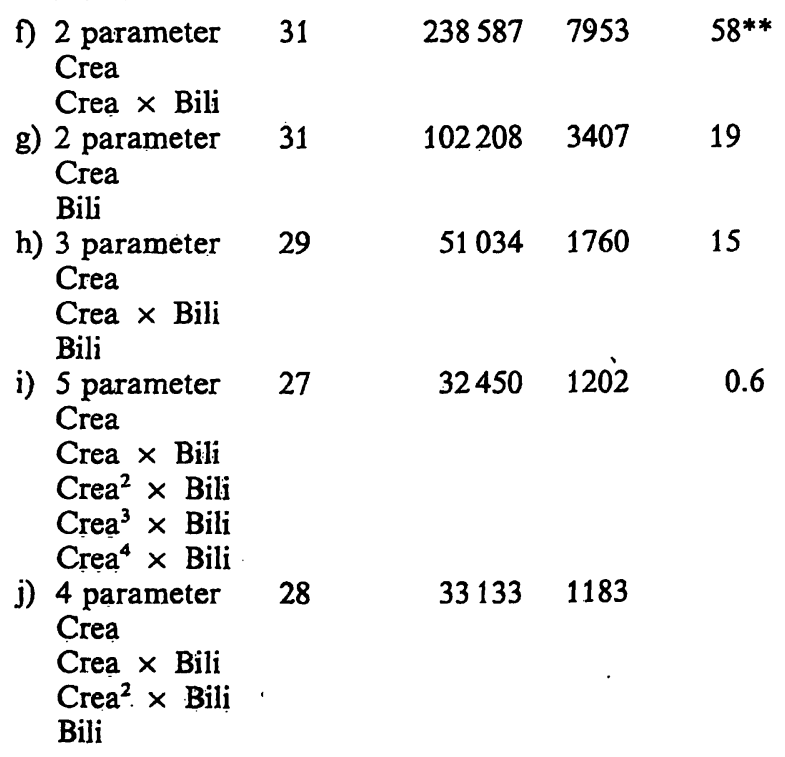

* Five parameter analyte-dependent model (e)

** Four parameter analyte-dependent plus analyte-independent model (j) where Crea' is the creatinine measured by the phenolaminophenazone peroxidase assay. Then we manually tested the models using StatView with forward and backward addition of terms. An automated stepwise regression neither minimizes the least squares fit nor is it a reasoned process. Moreover, programs for desktop computers usually do not generate higher ordered combination of variables or go back to look at terms which are insignificant early in the analysis. These reasons are critical to proper analysis. The routine interference and the best fitting models are tabulated in table 4. The models are ordered in descending order according to the size of the mean square of the residuals. To find the best fitting model with significant coefficients ( $p \leq 0.05$ ), we applied the F-test (the last column in tab. 4). We compared the model under consideration to what we considered the best model. The $90 \%$ confidence level is 1.6 .

\section{Discussion}

The basic mechanisms of interference are as follows:

(1) the interferent can either react with the reagents only or react with the sensor, like the product;

(2) it can react with the analyte or

(3) it can react with a product of the analyte and the reagents, interfering with the final interaction of the product and the sensor. The sensor is the detecting device, i.e. electrode or diode array. Only the first case is independent of the analyte concentration, the other two are dependent on it (8).

The standard linear regression models, that is simple regression, are shown in table 3. They appear adequate by conventional interpretation of interference, which assumes that the interference is independent of the analyte concentration. All the coefficients of determination, $\mathrm{r}^{2}$, are above 0.89 , which means that there is less than $5.6 \%$ probability that the association is due to chance. The slopes are significantly different from zero because the values for $p$ are less than or equal to 0.0085 ( $p=0.05$ is the cut off for significance). Therefore, each regression, in and of itself, would be considered acceptable by current standards. All previous studies of bilirubin interferences with the phenol-aminophenazone peroxidase creatinine method have assumed analyte-independence.

Examination of the slope in table 3 should be disconcerting. In true analyte-independent interference, the slopes of the regression should be the same over a wide range of analyte concentrations. This however, is not the case; these slopes vary significantly (e.g. 
comparing the first two slopes of the endpoint method, $t=3.09, v=8(v=$ number of degrees of freedom); therefore, significant at the 0.02 level of confidence). One way of verifying their significance is to regress these slopes against the creatinine concentration. Because the slopes are significantly different from zero, the interference has an analyte-dependent component. Figure 1 graphically reflects this analytedependence (slopes from table 3 ) and in addition shows the relationship to be non-linear with respect to creatinine concentration. Therefore, standard regression is insufficient to analyze the data because the analyte-dependence is missed. Multiple regression is the proper tool $(7-9)$.

Multiple regression analyses (tab. 4) provide greater insight than the standard regression. Complex models that involve multiple dependent variables and nonlinear terms are easily analyzed. Moreover, multiple regression uses the complete data set $(n=36)$, hence maximizing the degrees of freedom, whereas the standard regression reduces the data set to smaller sets thus reducing the degrees of freedom $(n=6)$. Table 4 lists the competing models according to the mean square of the residuals $\left(\mathrm{MS}_{\mathrm{r}}\right)$ in descending order. The best fitting models for the endpoint mode were the five parameter analyte-dependent interference (model e) and the six parameter mixed analyte-dependent and analyte-independent interference (model d). The Ftest revealed no statistical differences between these two models.

Two competing models also described the phenomena affecting the kinetic mode - one having strictly analyte-dependent terms (model i), and one having a mixture of analyte-dependent and analyte-independent terms (model j). These higher order complex models are more complicated than those we found with unconjugated bilirubin (11). These models listed above clearly fit the data better than do the conventional analyte-independent models for the equilibrium and kinetic methods (tab. 4, b and $\mathrm{g}$ ). In addition, the higher-order complex models ( $d, e, i$, and $j$ ), fit the data better than do the first-order analyte-dependent or mixed models (a, c, f, and h) by looking at the $\mathrm{F}$ ratio.

Although we cannot show that a statistical difference exists in the fit between these best competing models ( $d$ vs. e and i vs. j) for the endpoint and kinetic modes, respectively, they suggest different possible mechanisms. The mixture of analyte-dependent and analyteindependent terms suggests that at least two different mechanisms are at work. The complexity of these equations may indicate more than one site of interference of the same type of reaction.
Tab. 5. Polynomial curve fits for figure 1

\begin{tabular}{lllll}
\hline Model & p value $r^{2}$ & Sum & Mean & F ratio* \\
aCrea/ & for & of the & square \\
aBili $=$ & coeffi- & squares & of the \\
f(Crea, Bili) $)$ & cients & for the & residuals \\
& & residuals & \\
\hline
\end{tabular}

\begin{tabular}{|c|c|c|c|c|c|}
\hline \multicolumn{6}{|l|}{ Endpoint } \\
\hline a) $\begin{array}{l}b_{1} \text { Crea } \\
b_{2} \text { Crea }^{2}\end{array}$ & $\begin{array}{l}0.007 \\
0.020\end{array}$ & 0.977 & 0.00809 & 0.00270 & $\begin{array}{l}36 \\
\text { compared } \\
\text { to } 4 \text { th } \\
\text { order } \\
\text { model (c) }\end{array}$ \\
\hline b) $\begin{array}{l}b_{1} \text { Crea } \\
b_{2} \mathrm{Crea}^{2} \\
b_{3} \mathrm{Crea}^{3}\end{array}$ & $\begin{array}{l}0.195 \\
0.572 \\
0.894\end{array}$ & 0.987 & 0.00800 & 0.0040 & \\
\hline c) $\begin{array}{l}\mathrm{b}_{1} \text { Crea } \\
\mathrm{b}_{2} \mathrm{Crea}^{2} \\
\mathrm{~b}_{3} \mathrm{Crea}^{3} \\
\mathrm{~b}_{4} \mathrm{Crea}^{4}\end{array}$ & $\begin{array}{l}0.053 \\
0.069 \\
0.074 \\
0.075\end{array}$ & 1.000 & $0: 00011$ & 0.00011 & \\
\hline \multicolumn{6}{|l|}{ Kinetic } \\
\hline d) $\begin{array}{l}b_{1} \text { Crea } \\
b_{2} \mathrm{Crea}^{2}\end{array}$ & $\begin{array}{l}0.002 \\
0.007\end{array}$ & 0.991 & 0.00383 & 0.00128 & \\
\hline e) $\begin{array}{l}b_{1} \text { Crea } \\
b_{2} \mathrm{Crea}^{2} \\
b_{3} \mathrm{Crea}^{3}\end{array}$ & $\begin{array}{l}0.147 \\
0.824 \\
0.640\end{array}$ & 0.992 & 0.00333 & 0.00167 & \\
\hline f) $\begin{array}{l}\mathrm{b}_{1} \mathrm{Crea} \\
\mathrm{b}_{2} \mathrm{Crea}^{2} \\
\mathrm{~b}_{3} \mathrm{Crea}^{3} \\
\mathrm{~b}_{4} \mathrm{Crea}^{4}\end{array}$ & $\begin{array}{l}0.135 \\
0.203 \\
0.215 \\
0.206\end{array}$ & 0.996 & 0.00034 & 0.00034 & $\because$ \\
\hline
\end{tabular}

* Calculated only when the polynomial coefficients are significant

An alternative viewpoint of the data can help refine the selection of models. Figure 1 shows fitted lines through the slopes from simple regression analysis. The partial differential of the multiple regression equation should give the same coefficients as that of the polynomials used to fit the plot of the slopes. The curve that best fits the data can be found in the same manner as the best multiple regression equation. As shown in table 5 , for the endpoint analysis, the quartic curve (c) has borderline significant coefficients and the F-test shows that it is the best fit. Therefore the quartic polynomial supports the five parameter analyte-dependent endpoint (model e) in table 4 because the partial differential of the multiple regression equation gave the same coefficients as that of the polynomials used to fit the plot of the slopes ( $p>0.05$ ). However, we cannot make a case for or against (model d) because the bilirubin terms will not drop out in the first order derivative, hence not simplifying the equation to one variable. The best kinetic model is a quadratic equation (tab. 5-d) because all other models have insignificant coefficients. This polynomial curve fit analysis did not support the five parameter analytedependent kinetic model (tab. 4-i), and hence it can be eliminated. Therefore, we singled out the kinetic four parameter mixed analyte-idependent and analyteindependent model (tab. 4-j) as the model of choice. 
Tab. 6. Best fitting multiple regression models

\begin{tabular}{|c|c|c|c|c|}
\hline Variable/Model & Coefficient & $\mathrm{SE}_{\text {coer }}$ & t-value & $\mathrm{p}$ \\
\hline \multicolumn{5}{|c|}{ Endpoint method $(\mathrm{N}=36)$} \\
\hline \multicolumn{5}{|c|}{ Five parameter analyte-dependent (tab. 5 model e) } \\
\hline $\begin{array}{l}\text { Creatinine } \\
\text { Creatinine } \times \text { Bilirubin } \\
\text { Creatinine }^{2} \times \text { Bilirubin } \\
\text { Creatinine }^{3} \times \text { Bilirubin } \\
\text { Creatinine }^{4} \times \text { Bilirubin }\end{array}$ & $\begin{aligned} & 0.9991 \\
&-0.0020 \\
& 2.390 \times 10^{-6} \\
&-1.463 \times 10^{-9} \\
& 3.261 \times 10^{-13}\end{aligned}$ & $\begin{array}{l}0.0091 \\
0.0002 \\
4.5 \times 10^{-7} \\
3.52 \times 10^{-10} \\
8.62 \times 10^{-14}\end{array}$ & $\begin{array}{r}110 . \\
11 \\
5.3 \\
4.1 \\
3.8\end{array}$ & $\begin{array}{l}0.0001 \\
0.0001 \\
0.0001 \\
0.0003 \\
0.0007\end{array}$ \\
\hline
\end{tabular}

Intercept $9.9, \mathrm{r}^{2} 0.999$

$\begin{array}{lclrr}\text { Six parameter mixed analyte-dependent, analyte-independent (tab. 5, model d) } & & \\ \text { Creatinine } & 0.9834 & 0.0122 & 81 & 0.0001 \\ \text { Creatinine } \times \text { Bilirubin } & -0.000680 & 0.00012 & 5.5 & 0.0001 \\ \text { Creatinine }^{2} \times \text { Bilirubin } & 2.477 \times 10^{-7} & 3.40 \times 10^{-8} & 7.3 & 0.0001 \\ \text { Creatinine } \times \text { Bilirubin }^{2} & -3.233 \times 10^{-7} & 1.422 \times 10^{-7} & 2.3 & 0.0305 \\ \text { Bilirubin } & 0.4652 & 0.1186 & 3.9 & 0.0005 \\ \text { Bilirubin }^{2} & -0.00046 & 0.00017 & 2.7 & 0.0116\end{array}$

Intercept $12.2, \mathrm{r}^{2} 0.999$

Kinetic method $(\mathrm{N}=33)$

Four parameter mixed analyte-dependent, analyte-independent (tab. 5, model j)

Creatinine $0.9879 \quad 0.0134$

Creatinine $\times$ Bilirubin

Creatinine ${ }^{2} \times$ Bilirubin

$-0.00083$

$2.09 \times 10^{-7}$

0.0001

$5.38 \times 10^{-8}$

$-0.4524$

0.0918

Intercept 5.0, $\mathrm{r}^{2} 0.997$

What is important, is that the differences between the coefficients determined by the multivariate approach using the entire data set (tab. 4) and the polynomial fit to the slopes (tab. 5) are not significantly greater than zero $(p>0.05)$ for the analyte-dependent endpoint and the mixed analyte-dependent and -independent kinetic methods which mathematically validates our interpretation of the results. Table 6 list the models of choice derived from these analyses.

We have several cautionary notes about choosing and using a model. Creating more terms in the multiple regression equation does not necessarily mean a better fit or if a better fit that it is a better model. As can be seen by the MSr and SS values in table 4, these values fall with increasing number of terms. What has been omitted are the models with higher order terms that have insignificant coefficients. One has to be wary of "better fitting" higher order models as they may be fitting the noise in the data. Another point is that, one must always check the model against the experimental data and plot the residuals ((model calculation - data) vs. data). Performing this routine, we were puzzled that endpoint model (i) appeared to fit poorly. We found that a $20 \%$ round-off in the coefficient for $\mathrm{Crea}^{2} \times$ Bili, that was originally displayed as 0.000002 instead of 0.0000023901 , produced a negative bias as great as $1000 \mu \mathrm{mol} / 1$ ! This is because of the power of a higher order term times a very small coefficient can make a sizable contribution. Philosophically, the simplest model that fits the data is the most appropriate works very well in the kinetic model but there are subtleties which can be missed. In the endpoint method, we cannot discriminate between models (d) and (e), both fit the data equally well. However, mechanistically they suggest radically different pathways.

Comparing the four parameter mixed interference kinetic model with the equivalent endpoint model produced interesting results. There are no significant differences among the coefficients of the analyte-dependent terms $(p>0.05$ for both creatinine $\times$ bilirubin and creatinine ${ }^{2} \times$ bilirubin). This suggests that the analyte-dependent mechanisms do not change with time and that the any intermediates formed by the bilirubin-creatinine interferences are permanent. On the other hand, there is a significant difference in the analyte-independent term ( $p<0.05$ for bilirubin) that suggests transient analyte-independent mechanisms. Hence, the kinetic method is affected to a greater degree by analyte-independent effects than the endpoint.

Similar to unconjugated bilirubin, the synthetic conjugated bilirubin interferes by analyte-dependent 


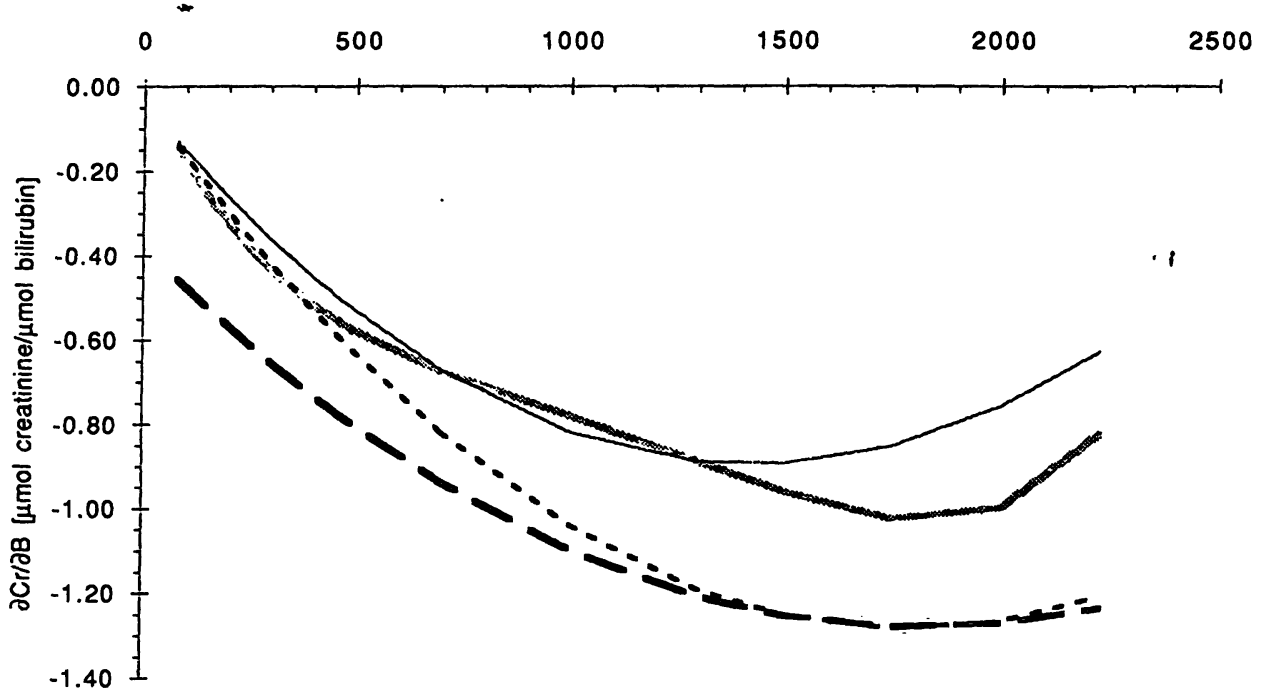

Creatinine $[\mu \mathrm{mol} / \mathrm{l}]$

Fig. 2. Predicted simple regression slopes, $\frac{\mathrm{dCrea}}{\mathrm{dBili}}$, for the experiments in which bilirubin and creatinine are varied.

Kinetic Mode: — Conjugated bilirubin Endpoint Mode: — Conjugated bilirubin
- Unconjugated bilirubin

Unconjugated bilirubin mechanisms as manifested by cross product terms in the multiple regression equations. Unlike unconjugated bilirubin, the synthetic conjugated bilirubin is also affected by significant analyte-independent mechanisms as manifested by a free bilirubin term in the multiple regression equations. Therefore, the two bilirubins react somewhat differently in the coupled phenol-aminophenazone peroxidase reactions. This begs the question - which bilirubin causes a larger interference? We compared the data from this experiment with that of unconjugated bilirubin (11). The data were not directly comparable as the values for bilirubin and starting creatinine differed. The residuals from comparing the models of predicted creatinine proved confusing to interpret in absence of a reference. Comparing the polynomials used to fit $\frac{\mathrm{dCrea}}{\mathrm{dBili}}$ for unconjugated bilirubin at various levels of starting creatinine (without interferent) versus that of conjugated bilirubin proved enlightening. Figure 2 compares the predicted $\frac{\mathrm{dCrea}}{\mathrm{dBili}}$ of conjugated bilirubin from the polynomial used to fit the slopes from simple regression analyses (tab. 2) with that used to describe the unconjugated bilirubin interference (11). The predicted simple regression slope for the kinetic mode is more negative for conjugated bilirubin than for unconjugated bilirubin below creatinine concentrations of approximately $1300 \mu \mathrm{mol} / \mathrm{l}$, especially at normal creatinine concentrations where the difference can be as high as $320 \%(-0.4496$ vs $-0.1397 \mu \mathrm{mol}$ creatinine $/ \mu \mathrm{mol}$ bilirubin). Hence the interference caused by conjugated bilirubin is greater than that by un- conjugated bilirubin. This also has been reported-by Franzini et al. (5) and Ihara et al. (6). However, lacking in their studies is what happens at the upper clinical ranges of creatinine. Figure 2 shows that in the kinetic mode the interference caused by both bilirubins not only increase but they converge to a slope of -1.26 ! We believe the same criticism can be said of work stating a lack of interference by delta bilirubin (15). As we have pointed out before, the whole range of possible laboratory values must be examined in interference studies $(7-9,11)$. The endpoint method behaves differently. Unconjugated and conjugated bilirubin presents similar interferences (fig. 2), until the concentration of creatinine is greater than 1300 $\mu \mathrm{mol} / \mathrm{l}$. Above this, the interference decreases more rapidly for the unconjugated bilirubin. Comparing the kinetic mode to the endpoint mode, the kinetic mode is more severely interfered with by either bilirubin. Further discussion as to why will occur when mechanisms are discussed.

Contour plots graphically illustrate how the measured creatinine is expected to vary over a range of true creatinine and conjugated bilirubin values (measured creatinine $=\mathrm{f}($ true creatinine, bilirubin)). In figure 3 , the contour lines are the true creatinine concentrations. The $\mathrm{x}$-axis is the observed creatinine. The values of the contours can be read off ${ }^{\prime}$ at zero bilirubin concentration. For example in figure 3 (bottom), the contour line labeled 83 is $83 \mu \mathrm{mol} / \mathrm{l}$ creatinine when there is no interference (i. e. no conjugated bilirubin). At $200 \mu \mathrm{mol} / \mathrm{l}$ conjugated bilirubin, the true creatinine of this contour would be approximately $180 \mu \mathrm{mol} / 1$ 


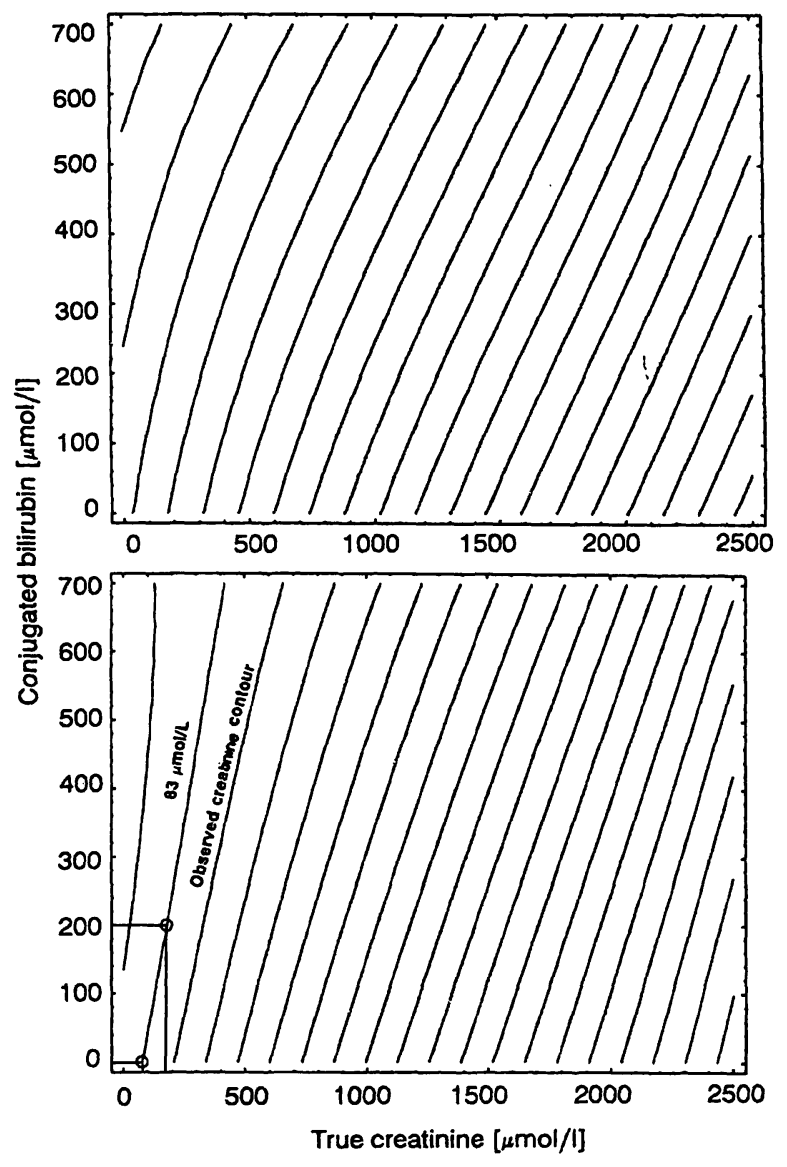

Fig. 3. Contour plots;

(top) represents the kinetic mode,

(bottom) represents the endpoint mode.

but would measure $83 \mu \mathrm{mol} / \mathrm{l}$. No interference would produce a family of vertical lines $\left(z=x_{n}\right)$. A simple negative analyte-dependent interference $(z=m x y)$ would lead to a family of lines with a positive slope (positive interference: $z=-m x y$ ). Our contour plots fall into neither category. The phenomenon is more complex and shows asymmetrical curves that demonstrate a non-linear analyte-dependent interference. The kinetic method, figure 3 (top), at creatinine concentrations below $1000 \mu \mathrm{mol} / \mathrm{l}$, the curves exhibit a bow progressively to the left as the creatinine approaches normal values, thus showing a system extremely sensitive to negative interference at high bilirubin concentrations. The contours show that a single slope, such as that produce by simple regression anaylsis, would inadequately describe the interference over even a small range of creatinine concentrations. As the concentrations of creatinine increase, the curves straighten out to the point where a linear approximation looks good over the entire range of bilirubin. This result reflects the effects of the higher order terms of the multiple regression equation. The change in slope to a shallower angle reflects an increasing negative interference. Figure 3 (bottom), the endpoint method combined analyte-dependent and
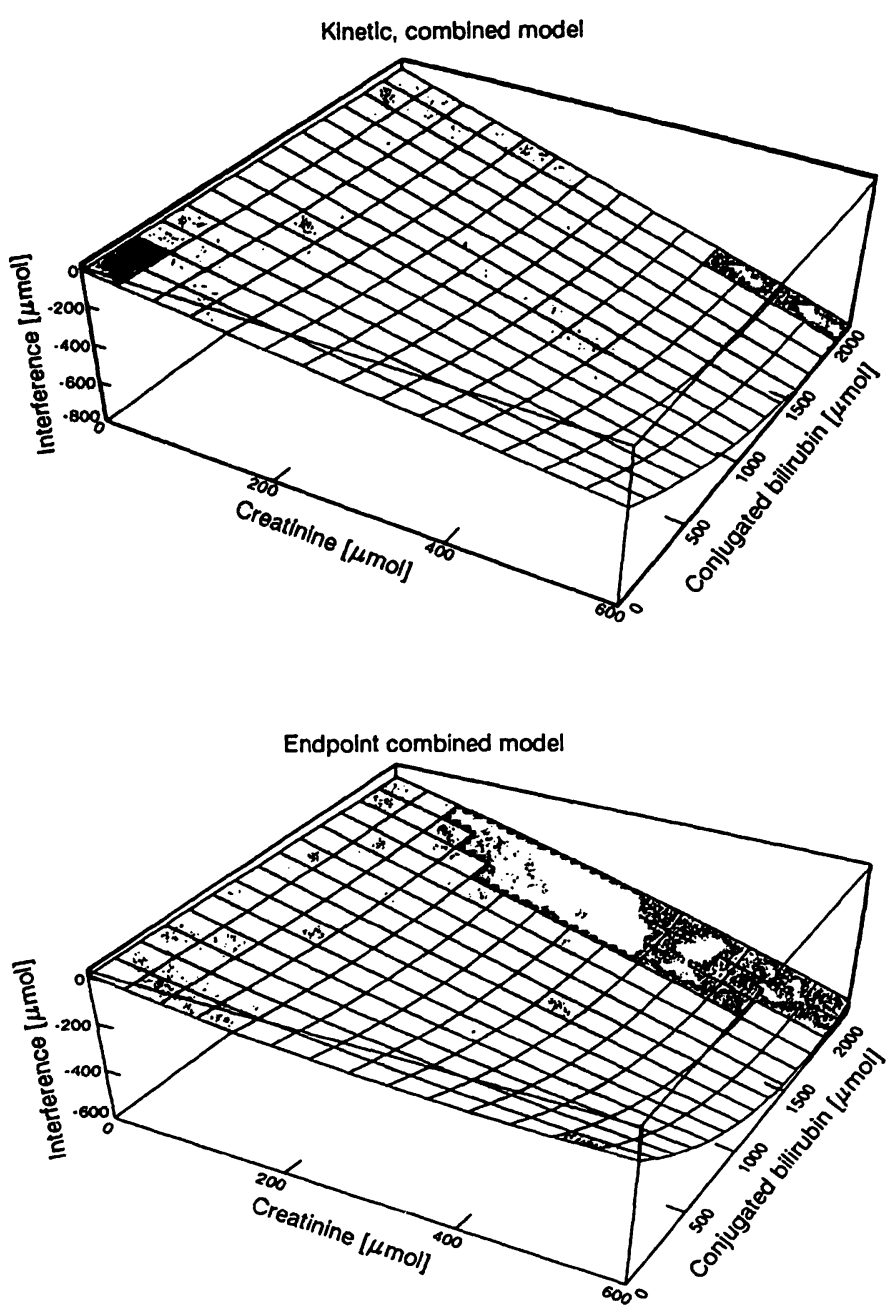

Fig. 4. Surface response plots;

(top) represents the kinetic mode,

(bottom) represents the endpoint mode.

analyte-independent model (model (h)) has noticably steeper slopes than figure 3 (top), which denotes less interference. The curve on the extreme left is interesting. At high concentrations it is a vertical line, indicating no increasing interference with increasing bilirubin (i. e. tab. 2, second line of data). This may mean a saturation of the interfering mechanism. The contour plots provide a graphical explanation for the initially discrepant information seen in table 3 and figure 1 where the simple linear regression models indicate a change in slopes from lower creatinine concentrations to higher ones.

Response surface curves or surface plots are another useful manner to see the effect of interferences. Figure 4 shows the three dimensional surface plots for the modeled responses in the kinetic and endpoint reactions. The kinetic method (4 (top)) shows that at normal concentrations of creatinine, bilirubin produces a near linear negative response. This suggests that the analyte-independence is the predominate mechanism of action in this range. However, as the domain is extended, the surface curves along the cre- 
atinine axis which denotes the analyte-dependent mechanisms coming into play. The endpoint method (4 (bottom)) shows a curve when bilirubin increases when the creatinine is at normal levels. Hence, analyte-dependent mechanisms are either predominating or significantly contributing to the interference. Support for this, can be seen in table 3 , the first slope for the endpoint method only has an $r^{2}$ of 0.893 compared to 0.970 or better for the other levels of creatinine. The greater curvature of the curve along the creatinine axis compared to the kinetic method show that the analyte-dependent mechanisms are stronger in the time period for the endpoint method than in the kinetic method. However, overall, the kinetic method is more negatively affected than the endpoint method as can be seen from the scale of the interference axis. In contrast to the multivariate approach we describe, each simple linear regression will only yield one line of the surface response per experiment. Even if many experiments were performed, one would have to interpolate between lines which can be marred by large errors if the non-linearities are large. As one can see, this is not a problem with the mulivariate approach.

Clinically, the derived polynomial equation allows one to estimate the magnitude of the interference, as well as to be able to solve for the true creatinine concentration despite the conjugated bilirubin interference. When equations for both unconjugated (11) and conjugated bilirubin are evaluated simultaneously, a better estimation of the patient may result. However, at this time, the effect of delta bilirubin remains unknown.

Although the derived models are correlative, the terms from the multiple regression suggest overall mechanisms that are based on the general mechanisms of interference (8). The analyte-independent term, the freestanding bilirubin term, $B$, can represent many possibilities. Classically, a spectral interference comes to mind, but if that is case it is not constant as kinetic method is affected to a greater degree. Bilirubin can react reversibly or irreversibly with the reagents causing greater interference during the time of the kinetic measurements. For example, bilirubin directly or indirectly may tie up any of the enzymes. For example, bilirubin may inhibit creatinine's reaction with creatininase or any of the subsequent reactants with the succeeding enzymes by temporarily binding to the enzymes. It may be oxidized by oxygen which is a reagent in the third step, thus slowing the oxidation of sarcosine until the bilirubin is consumed. Any of the Trinder reagents may also react either permanently or reversibly with bilirubin and would be considered analyte-independent mechanisms. The bilirubin ${ }^{2}$ term in the six parameter endpoint model may suggest the rare possibility of autoreaction, whereby bilirubin reacts with itself before partaking in the other discussed mechanisms.

The analyte-dependent or cross-product terms, creatinine $\times$ bilirubin, creatinine ${ }^{2} \times$ bilirubin etc., imply a reaction either with the analyte directly or a product of the analyte and reagents. These can be side reactions to the ususal coupled reaction in the phenolaminophenazone peroxidase method. A long-lived intermediate product formed among the interferent, the substrate, and the enzyme. For example, bilirubin can form a complex with one to two creatinine molecules and creatinase. Such a model would also account for the creatinine $e^{2} \times$ bilirubin term and higher terms. Alternatively, bilirubin could react with more than one enzyme complex or the various intermediate products. Not all reactions need cause a negative interference but the sum of all the contributions will be negative. One has to consider the possible reaction of bilirubin with intermediate products such as sarcosine or more likely, hydrogen peroxide. A short-lived intermediate is one mechanism that would account for the greater interference in the kinetic mode (fig. 2). At higher concentrations of creatinine, both the reduction of the interference in the endpoint mode and the flat interference in the kinetic mode may be caused by the analyte or its product intermediates, overtaking the interferent's effect. It is possible that side reactions of bilirubin with the other reactants would contribute to the cross-product terms in the multiple regression equations. Bilirubin interferes with several peroxidase-coupled assays (19-22) which include cholesterol, uric acid and triglycerides. Bilirubin may compete with the Trinder reagent for hydrogen peroxide. If this is the case, then it is the point where bilirubin interferes with all these assays and interferences similar to what we describe for the creatinine phenolaminophenazone peroxidase method can be expected. Witte proposed that bilirubin destroys the intermediate or forms a long-life intermediate of the peroxidase reaction (22).

Complex analyte-dependent/independent interferences have terms that are non-linear, meaning terms with a power different from one. The non-linear term implies either positive or negative feedback or cooperation. Positive feedback means that a chemical which is either native to the reaction or exogenous to it will enhance the reaction. Examples include free radical reactions, enhanced binding at a second binding site after the first site is occupied such as oxygen to haemoglobin, and of course, enzymatic reactions. Autocatalytic reactions are a suibclass of positive feedback. Negative feedback will produce a decreased 
reaction rate. Alternatively, the multiple regression equation represents the first few terms in a twovariable Taylor's series for a yet to be determined non-linear function that describes the relationship over the entire range and provides the mathematical basis for a mechanism. We are exploring methods to fit equations of the surface responses produced by the experiment to produce a less cumbersome mathematical expression. The current analyses are similar to thermodynamic studies in that we studied only the endpoints of each reaction mode. Analysis of the kinetic rate constants for each set of reactions can be used to isolate the points of interference as well as to define the true mechanisms (23).

In conclusion, we have demonstrated that bilirubin not only interferes negatively with the creatinine phenol-aminophenazone peroxidase methodology but it does so in an analyte-independent and an analytedependent manner. Furthermore, in the kinetic method, the analyte-dependence is second-order nonlinear relationship with respect to creatinine, thirdorder overall. The interference in the endpoint method can be described by two possible models. One is a fourth-order analyte-dependent model and the other is a complex combined analyte-independent, analytedependent model in which the highest terms are second order with respect to creatinine and to bilirubin. We may be the first to demonstrate such complex non-linear terms that may denote positive and negative feedback reactions as underlying mechanisms of interference. The two modes of measurement allowed us to study an interfering reaction which changes with time. The analyte-dependent component terms are similar to that seen with unconjugated bilirubin (11).
The routine analysis of interferences, that is, simple regression, failed to yield an adequate analysis. Conjugated bilirubin affected the kinetic method more than the endpoint method. We also present evidence that conjugated bilirubin interferes the kinetic method more than unconjugated bilirubin for creatinine concentrations less than $1300 \mu \mathrm{mol} / 1$ but converge to a slope of $-1.2 \mu \mathrm{mol}$ creatinine per $\mu \mathrm{mol}$ bilirubin. Unconjugated and conjugated bilirubin present similar interferences in the endpoint method at concentrations of creatinine below $1300 \mu \mathrm{mol} / \mathrm{l}$. Above this, the interference decreases more rapidly for the unconjugated bilirubin.

With the advent of desktop computers, multiple regression analysis is a facile operation and can provide a more accurate estimation of the relationship involved. Simple linear regression is an inadequate tool to study interferences because it shows only one line of a surface response which is deceiving when there is an analyte-dependent mechanism involved. This approach should advance the analysis of interferences and allow clinical chemists and chemical pathologists to make sense out of comparisons with other studies that may produce different slopes because of the different range studied, and yet accurately describe the relationship over a small interval. It would be desirable for instrument manufacturer's to provide similar analyses on interferences.

\section{Acknowledgement}

The authors are grateful to Dr. George Hutchinson from the NIH Division of Computer Research and Technology for producing the surface response plots.

\section{References}

1. Weber, J. A. \& Zanten, A. P. (1991) Interferences in current methods for measurements of creatinine. Clin. Chem. 37, 695-700.

2. Margrey, M., Margrey, K., Bruns, D. E., Boyd, J. C., Fortier, G. A., Renoe, B. W. \& Savory, J. (1984) Enzymatic assay for creatinine with fixed-time kinetics on a centrifugal analyzer. Ann. Clin. Lab. Sci. 14, 298-303.

3. Guder, W. G. \& Hoffman, G. E. (1986) Multicentre evaluation of an enzymatic method for creatinine determination using a sensitive colour reagent. J. Clin. Chem. Clin. Biochem. 24, 889-902.

4. Lindback, B. \& Bergman, A. (1989) A new commercial method for the enzymatic determination of creatinine in serum and urine evaluated: Comparison with a kinetic Jaffé method and isotope dilution-mass spectrometry. Clin. Chem. 35, 835-837.

5. Franzini, C., Morelli, A. M. \& Cattazzo, G. (1991) Use of a synthetic soluble bilirubin derivative to assess interference in creatinine measurements. Clin. Chem. 37, 236-238.

6. Ihara, H., Nakamura, H., Yoshida, M. \& Wu, T. W. (1992) Effects of bilirubin covalently attached to albumin on measurement of serum creatinine. [letter]. Clin. Chem. 38, $321-$ 322.

7. Kroll, M. H. \& Chesler, R. (1992) Rationale for using multiple regression analysis with complex interferences. Eur. J. Clin. Chem. Clin. Biochem. 30, 415-424.

8. Kroll, M. H., Ruddel, M. E., Blank, D. W. \& Elin, R. J. (1987) A model for the assessment of interference. Clin. Chem. 33, 1121-1123.

9. Kroll, M. H. (1991) Analyte-dependent interference and multi-interference. Deutsche Gesellschaft Klin. Chem. Mitteilungen $22,13-20$.

10. Letellier, G. (1980) How does drug interference relate to quality? In: Quality Assurance in Health Care: A Critical Appraisal of Clinical Chemistry. AACC, Washington, D. C., pp. $135-169$.

11. Eng, C. D., Delgado, R. \& Kroll, M. H. Multivariate analysis of the analyte-dependent bilirubin interference in 
the creatinine phenol-aminophenazone peroxidase method. (submitted)

12. Jirsa, M., Vecerek, B. \& Ledvinas, M. (1956) Di and monotaurobilirubin similar to a directly reacting form of bilirubin in serum. Nature 177, 895 (letter).

13. Ostrow, Donald, J., Murphy, J. \& Nancy, H. (1977) Isolation and properties of conjugated bilirubin from bile. Biochem. J. 120, 322-327.

14. Steinbrink, C. F. \& Blumenfeld, T. A. (1977) An evaluation of total serum conjugated bilirubins using two different diazosulfanile acid methods. Clin. Chem. 23, 1128 (abstract).

15. Balistreri, W. F. \& Shaw, L. M. (1986) Liver function. In: Textbook of Clinical Chemistry (Tietz, N. W., ed.) pp. 1373-1433, WB Saunders Co., Philadelphia.

16. Crocker, H., Shephard, M. D. S. \& White, G. H. (1988) Evaluation of an enzymatic method for determining creatinine in plasma. J. Clin. Pathol. 41, 576-581.

17. Benson, P. \& Apple, F. (1986) Evaluation of the BMD enzymatic creatinine PAP method on a Cobas-Bio. Clin. Chem. 31, 1100 (Abstract).

18. Draper, N. \& Smith, H. (1981) Applied Regression Analysis 2nd Edition, pp. 294-351, 412-422. John Wiley \& Sons, Inc., New York.
19. Aoki, Y., Ihara, H., Nakamura, H., Aoki, T. \& Yoshida, M. (1992) Effects of serum bilirubin on determination of uric acid by the uricase-peroxidase coupled reaction. Clin. Chem. 38, 1350-1352.

20. Spain, M. A. \& Wu, A. H. B. (1986) Bilirübin interference with determination of uric acid, cholesterol, and triglycerides in commercial peroxidase-coupled assays, and the effect of ferrocyanide. Clin. Chem. 32, 518-521.

21. Hullin, D. A. \& McGrane, M. T. G. (1991) Effect of bilirubin on uricase-peroxidase coupled reactions. Implications for urate measurement in clinical samples and external quality assessment schemes. Ann. Clin. Biochem. 28, $98-100$.

22. Witte, D. L., Brown, L. F. \& Feld, R. D. (1978) Effects of bilirubin on detection of hydrogen peroxide by use of peroxidase. Clin. Chem. 24, 1778-1782.

23. Kroll, M. H. \& Elin, R. J. (1983) Mechanism of cefoxitin and cephlothin interference with the Jaffé method for creatinine. Clin. Chem. 29, 2044-2048.

Clifford D. Eng, M. D.

Rosario Delgado

Martin H. Kroll, M. D.

Clinical Pathology Department

Warren G. Magnuson Clinical Center

Building 10, Room $2 \mathrm{C} 407$

National Institutes of Health

Bethesda, MD 20892

USA 